\title{
La reciente evolución de la estrategia antiterrorista, test de la estrategia global de seguridad de la UE*
}

\section{Current evolution of the counterterrorism strategy: assessment of the European Common Security and Defense Policy}

\author{
Consuelo RAMÓN CHORNET \\ Catedrática de Derecho Internacional Público y Relaciones Internacionales \\ Universidad de Valencia \\ consuelo.ramon@uv.es
}

Abstract: This article focuses in the analysis of the European Common Security and Defence Policy's evolution, specifically taking the European Global Agenda presented by the High Representative F. Mogherini in the European Council of June 2016 as the starting point. This strategy includes as a priority the fight against terrorism.

Keywords European security / European Global Strategy of Security and Defence / European Union's measures against terrorism
Résumé: Cet article examine l'évolution récente de la politique européenne commune en matière de sécurité et de défense au cours des 15 mois qui se sont écoulés depuis la présentation de l'agenda global européen par F.Mogherini prés du Conseil Européen (juin 2016). Plus précisément, ce qui est prioritaire dans cette stratégie de sécurité, c'est á dire, la lutte contre la menace du terrorisme international.

Mots clés: Stratégie européenne globale de sécurité et de défense, Lutte européenne face au térrorisme, PCSD, PESD

Sumario: 1. UNA POLÉMICA QUE NO CESA: EL MODELO DE POLITICA EUROPEA DE SEGURIDAD Y DEFENSA Y LA ESTRATEGIA GLOBAL DE 2016. 2. UN CONTEXTO MARCADO POR LA AMENAZA TERRORISTA. 3. PRINCIPALES HITOS DE LA EVOLUCIÓN DE LA ESTRATEGIA GLOBAL EN 2017. EN PARTICULAR, EN MATERIA DE LUCHA ANTITERRORISTA. 3.1. El desarrollo de la estrategia global de seguridad y defensa en 2017. 3.2. Los avances en materia de lucha europea contra el terrorismo en 2017. 4. SOBRE LA ESTRATEGIA ANTITERRORISTA DEL EJE FRANCO-ALEMÁN. 5. PRINCIPIOS Y BUENAS PRÁCTICAS EN LA ESTRATEGIA ANTITERRORISTA, COMO PRIORIDAD DE LA POLÍTICA DE SEGURIDAD Y DEFENSA EUROPEAS.

* Este artículo se inscribe en el Proyecto I+D (Ref.: DER2014-57671-R): La estrategia de Seguridad nacional de España. Un enfoque geográfico (IP: Javier Roldán) y en el Proyecto de Excelencia DER2015-69273 RED (dirigido por Pablo A. Fernández). 


\section{UNA POLÉMICA QUE NO CESA: EL MODELO DE POLÍTICA EUROPEA DE SEGURIDAD Y DEFENSA Y LA ESTRATEGIA} GLOBAL DE 2016

unque la definición de una política europea común de seguridad y
defensa en sentido estricto es algo reciente, es un lugar común la
afirmación de que el fracaso inicial del proyecto político europeo se debió precisamente a la falta de acuerdo sobre el alcance y características de una posible política europea de defensa. En efecto, se repite que fue la oposición frontal de Francia a su creación (un Tratado en el que se trabajaba ya en 1951, junto al Tratado de una comunidad política europea) la razón fundamental de que el propio proyecto europeo se quedara reducido al ámbito económico-comercial ${ }^{1}$. Desde entonces, ha habido que esperar hasta 2007 , con el Tratado de Lisboa ${ }^{2}$, para recuperar la idea específica de una «Europa de la defensa», ahora en el marco de la Política Exterior y de Seguridad europea (que incluye la PCSD). La cuestión es si se ha producido un avance significativo en este objetivo.

Para responder a esta pregunta, en las páginas siguientes trataré de examinar algunos desarrollos recientes que se han producido a lo largo de 2017, tomando como punto de partida claro está, la Estrategia global para la política exterior y de seguridad de la Unión Europea ${ }^{3}$, presentada por la Alta Represen-

1 Así lo acaba de recordar la editorial del monográfico sobre «Europa de la Defensa» en el número 275 (octubre 2017) de la revista Temas (Fundación Sistema) «Hacia una política de seguridad y defensa europea», que insiste en considerar ese rechazo francés como el punto de inflexión que determinó la reducción del proyecto europeo a su dimensión de comunidad económica, además de costarle a Jean Monnet la presidencia de la CECA.

2 Así se afirma ya desde el Preámbulo del propio TUE: «RESUELTOS a desarrollar una política exterior y de seguridad común que incluya la definición progresiva de una política de defensa común que podría conducir a una defensa común de acuerdo con las disposiciones del artículo 42, reforzando así la identidad y la independencia europeas con el fin de fomentar la paz, la seguridad y el progreso en Europa y en el mundo». Todo ello encuentra concreción y desarrollo en el título V, capítulo 2, sección 2, artículos 42 a 46, así como en los Protocolos 1, 10 y 11 y en las Declaraciones 13 y 14.

3 El texto del documento de Estrategia global europea de seguridad y defensa, presentado con el título Una visión común, una estrategia conjunta, una Europa más fuerte, puede consultarse en el sitio web https://europa.eu/globalstrategy/sites/globalstrategy/files/eugs_es_version.pdf. La Estrategia arranca con esta afirmación: «Los objetivos e incluso la propia existencia de nuestra Unión están en entredicho... nuestra región, en su extensión más amplia, es cada vez más inestable e insegura. Las crisis dentro y fuera de nuestras fronteras están afectando directamente a la vida de nuestros ciudadanos» (p.3). Como se recordará, el modelo que preconizaba en 2016 
tante Federica Mogherini en el Consejo Europeo de 28/29 de junio de 2016, una ambiciosa inicitiva largamente elaborada y en cuyo seno se propone de nuevo una política común europea, global y autónoma de defensa y de seguridad $^{4}$.

En algunos trabajos anteriores he tratado de analizar ese documento de estrategia global, en términos de una propuesta de un modelo autónomo de la UE en materia de seguridad y defensa ${ }^{5}$. En las páginas que siguen me centraré, de modo más concreto, en la evolución más reciente de las respuestas adoptadas por la UE en relación con la prioridad señalada en ese documento, la lucha contra el terrorismo internacional, señalado como la amenaza más grave para la seguridad europea ${ }^{6}$. Creo que esa evolución demuestra que, contra todo pronóstico, algo se ha movido a lo largo de estos meses. Aún más, que cabe ofrecer algunos argumentos que señalan hacia dónde puede encaminarse esa nueva política europea y muy en particular, en lo que se refiere al mencionado objetivo prioritario de la Política común de seguridad y defensa $\left(\mathrm{PCSD}^{7}\right)$, parte muy relevante de la Política Exterior de la UE, a partir de algunos cambios que se pueden apreciar en elementos clave del contexto en el que nos encontramos hoy, al filo del último trimestre de 2017.

la Alta Representante Mogherini propone adoptar una estrategia que, al tiempo que global, permita construir lo que se denomina «una visión compartida y una acción común» que haga posible «una autonomía estratégica» de la UE.

4 Sobre la evolución de esa estrategia europea y los antecedentes que llevan a la propuesta de la Alta Representante en junio de 2016 puede consultarse el ensayo en DE CARLOS IZQUIERDO, J., «La nueva Estrategia de seguridad Europea 2016», IEEE, Documento Marco de 30 de septiembre de 2016.

5 Cfr. puede verse, RAmón ChORnet, C., «La contribución de España a las prioridades de la estrategia global para la Política Exterior y de Seguridad de la UE del Brexit», en F.J. Roldán (coord.), La seguridad nacional de España: un enfoque geoestratégico, Tirant lo Blanch, Valencia, 2017.

6 Conviene tener presente que de los cinco objetivos prioritarios de la PESC (la seguridad de la Unión; la resiliencia estatal y social de los vecinos orientales y meridionales de la Unión; la concepción de un enfoque integrado en relación con los conflictos; el establecimiento de órdenes regionales de cooperación y, finalmente, una gobernanza mundial para el siglo XXI), aquel que centra los mayores esfuerzos es el primero, y en ese marco de la prioridad de la seguridad, la lucha contra el terrorismo constituye la prioridad de prioridades.

7 Antes PESD, Política europea de seguridad y defensa. Sobre la evolución de la PESD a la PCSD y sus principales retos, remito a la monografía Dumoulin, A. y Gros-VerHEYDE, N., La politique européenne de sécurité et de défense commune (Parce que l'Europe vaut bien une défense), Du Villard, Bruxelles, 2017, que incluye un interesante prefacio escrito por la Alta Representante, Federica Mogherini. 


\section{UN CONTEXTO MARCADO POR LA AMENAZA TERRORISTA}

Sería erróneo hablar de un cambio en el contexto internacional en los últimos doce meses, en el que se enmarca la estrategia de lucha contra el terrorismo, entendido como amenaza no ya internacional, sino global. Lo que es obligado reconocer es que la percepción de la amenaza del terrorismo internacional no ha dejado de acrecentarse en este año y de esa manera se confirma lo que, expresamente, desde el año 2015, la UE considera la amenaza más grave en términos de seguridad ${ }^{8}$, aunque la noción de seguridad global -y, desde luego, la de seguridad humana- obliga a ampliar el elenco de amenazas y -lo que es sustancialmente distinto- el de retos.

Con todo, los atentados terroristas a lo largo de 2016, que no han dejado de agravarse en 2017, evidencian que el terrorismo internacional constituye el mayor de los retos para la seguridad europea ${ }^{9}$. Por eso, la UE ha tratado de poner en marcha un abanico de medidas que comienza por la mejora de la colaboración en los servicios de información e inteligencia, e incluye entre otras la lucha contra la radicalización y la cooperación con los países en los que la violación de los derechos humanos o la ausencia de indicadores de desarrollo pueden generar la aparición de organizaciones terroristas que pongan en jaque la estabilidad de esos países y supongan riesgo para la $\mathrm{UE}^{10}$.

8 COM (2015) 185 final, de 28 de abril de 2015. Puede consultarse en http://eur-lex.europa.eu/ legal-content/ES/TXT/PDF/?uri=CELEX:52015DC0185\&from=ES

9 A ese respecto, es muy útil el Informe de Europol 2017, European Union. Terrorism Situation and Trend Report https://www.europol.europa.eu/activities-services/main-reports/eu-terrorismsituation-and-trend-report-te-sat-2017

10 Así lo expone el apartado 3.1 del mencionado documento de Estrategia cuando aborda específicamente la amenaza del terrorismo: «Se han perpetrado grandes atentados terroristas en suelo europeo y otros lugares. Es fundamental una mayor solidaridad y mayores inversiones en materia de lucha contra el terrorismo. Por ello, queremos fomentar un mayor intercambio de información y mayor cooperación en materia de inteligencia entre los Estados miembros y las agencias de la UE. Ello implica compartir descripciones y alertas sobre el extremismo violento, las redes terroristas y los combatientes terroristas extranjeros, así como el control y la supresión de contenidos ilícitos de los medios de comunicación. Al mismo tiempo, la UE apoyará la rápida recuperación de los Estados miembros en caso de ataques, mediante un refuerzo de la seguridad de los suministros, la protección de las infraestructuras críticas y la potenciación del marco voluntario de gestión de cibercrisis. Se intensificarán los trabajos sobre educación, comunicación, cultura, juventud y deporte para luchar contra el extremismo violento. Trabajaremos en la lucha contra la radicalización, ampliando nuestras asociaciones con la sociedad civil, los agentes sociales, el sector privado y las víctimas del terrorismo, así como mediante el diálogo intercultural e interreligioso. Y lo más importante, la UE estará a la altura de sus valores a nivel interno y externo: es el mejor antídoto que tenemos contra el extremismo violento. También vamos a seguir 
El curso posterior de los acontecimientos, como recordaba, ha venido a confirmar esa preocupación. A lo largo de 2017 hemos vivido terribles muestras de ello en seis diferentes atentados, con medio centenar de muertos y un número muy elevado de heridos ${ }^{11}$, atentados caracterizados en buena medida por su ejecución mediante diferentes manifestaciones de lo que se ha dado en llamar, a falta de una categoría más precisa, el terrorismo low-cost, es decir, acciones las más de las veces individuales, que utilizan medios al alcance de cualquiera (automóviles, autobuses, camiones), armas blancas o en todo caso armamento poco sofisticado, obra las más de las veces de fabricación casera, lo que produce también que sus autores puedan ser víctimas de la preparación, como sucedió en el caso de los terroristas que elaboraban explosivos en la localidad tarraconense de Alcanar, antes de cometer sus atentados en Barcelona y Cambrils. En todo caso, me parece importante dejar ya sentado un hecho que debiera servirnos para relativizar la tentación de presentar a la UE como el blanco preferente de la acción del terrorismo internacional. Sólo por referirnos al acontecimiento más reciente en el momento de redactar estas páginas, los dos atentados de la milicia terrorista yihadista $\mathrm{Al}$ Shabab con camiones bomba en Mogadiscio (Somalía), el 14 de octubre de 2017, se han cobrado más de 350 muertos $^{12}$.

Sentada esa matización, me parece necesario añadir otras dos. La primera, que ya he avanzado antes, es que si aceptamos una noción de seguridad humana, global, deberíamos evitar que la necesaria dimensión policial/militar de seguridad provoque un deslizamiento de la noción de seguridad hacia la de defensa y aun monopolice la construcción de una política europea de seguri-

desarrollando una cooperación antiterrorista que sea respetuosa de los derechos humanos en África del Norte, Oriente Próximo, los Balcanes Occidentales y Turquía, entre otros, y a trabajar con socios de todo el mundo para poner en común las prácticas idóneas y desarrollar programas conjuntos en materia de lucha contra el extremismo violento y la radicalización».

11 El primer atentado tuvo lugar en Londres, el 22 de marzo de 2017, cuando un hombre arrolló con su vehículo a varias personas en el puente de Westminter y acuchilló a un policía a las puertas del Parlamento, cobrándose la vida de tres personas, al que siguió el de los Campos Elíseos en París, el 20 de abril, que costó la muerte por disparos a un gendarme, hasta el masivo atentado con explosivos en Manchester, el 22 de mayo con 22 muertos en un concierto en el Manchester Arena. El cuarto se produjo de nuevo en Londres, el 3 de junio, de nuevo por atropello seguido de apuñalamientos que se cobraron 7 víctima mortales. El 17 de agosto, en Barcelona y Cambrils, los atropellos y apuñalamientos costaron 15 muertos. Como atentado terrorista se identifica también el ataque con arma blanca en Turku, el 18 de agosto de 2017, que costó 2 muertos y 8 heridos.

12 Cfr. la información sobre esos atentados ofrecida, por ejemplo, por The Guardian: https://www. theguardian.com/world/2017/oct/16/mogadishu-bombing-al-shabaab-behind-deadly-blastofficials-say (consultada 16 octubre 2017). 
dad y defensa ${ }^{13}$. La segunda, la necesidad de ser exquisitamente escrupulosos con el riesgo que siempre supone el ceder a la tentación de abordar esa amenaza real en términos de estado de excepción, que afecta a la garantía eficaz de los derechos fundamentales. El ejemplo de Francia es relevante, como trataré de mostrar después, al examinar los elementos que pueden permitir albergar algún optimismo sobre la evolución de la política europea de seguridad y defensa en punto a la estrategia antiterrorista.

Debe apuntarse también que quienes señalaron el posible impacto negativo de la salida del Reino Unido en el diseño de la lucha de la UE contra la amenaza del terrorismo internacional, han encontrado en estos meses un contundente desmentido. En efecto, después de una tan inaceptable como precipitada amenaza de la premier May a propósito del papel del Reino Unido en tareas de seguridad europea frente al terrorismo que, para su desgracia, fue enunciada muy poco antes del atentado del Manchester Arena, May tuvo que rectificar y la implicación del Reino Unido en tareas básicas de la lucha antiterrorista es hoy inequívoca, como no podía ser de otra manera.

Quizá lo que ha venido a confirmarse en estos meses son algunas tendencias en las relaciones internacionales que complican el objetivo de configurar la estrategia europea antiterrorista como estrategia global. Pocas exposiciones más claras de este complejo panorama internacional como la que ofrece el documento oficial Révue Stratégique de la Défense et de la Sécurité Nationale $2017^{14}$, del Gobierno Macron, recientemente publicado. El apartado $2^{\circ}$ de la Synthése de ese documento ofrece, a mi juicio, una excelente descripción,

13 Como se puso de manifiesto con ocasión de los atentados en Barcelona y Cambrils, en los que se planteó un debate a veces agrio sobre la cooperación eficaz de los servicios oficiales y de inteligencia (a propósito de mensajes intercambiados entre los Mossos d'Esquadra, los servicios policiales y de inteligencia integrados en el Centro de Inteligencia contra el Terrorismo y el Crimen Organizado (CITCO) y los servicios policiales belgas y de inteligencia de los EEUU), esta es una cuestión clave, ante todo dentro de cada Estado, pero también entre los Estados de la UE. Hay que recordar que, muy poco después de los atentados de 2001, la UE creó la red ATLAS, para mejorar la cooperación de unidades especiales de policía de los Estados miembros de la UE, especializadas en la lucha contra el terrorismo y la delincuencia.

14 Révue Stratégique de la Défense et de la Sécurité Nationale 2017, disponible en el sitio web de la DGRIS (Diréction Générale des Relations Internationales et de la Stratégie), http://www.defense. gouv.fr/dgris/la-dgris/evenements/revue-strategique-de-defense-et-de-securite-nationale-2017 (consultado el 15 octubre 2017). Es imposible dejar de apreciar la relación entre ese documento y el muy comentado discurso del Presidente Macron sobre un nuevo modelo para el proyecto de la UE, «Une Europe souveraine, unie et democratique», pronunciado el 26 de septiembre de 2017 en el gran anfiteatro de La Sorbonne, que se puede consultar en el Cuaderno 445 (2 de octubre de 2017) de la serie Questions d'Europe, de la Fondation Robert Schuman, bajo el título L'Europe 
bajo el rótulo «Un environnement stratégique instable et incertain», donde se explican con toda claridad el incremento de la inestabilidad e incertidumbre en esas relaciones internacionales como consecuencia de la emergencia de políticas de hechos consumados, de la competencia cada vez más feroz por los recursos energéticos y a ese propósito, el papel dominante de China, con su toma de posición en el continente africano, por ejemplo, es un factor que no se puede desdeñar. Directamente relacionado con el recrudecimiento de la amenaza terrorista es la creciente impugnación de las normas internacionales relativas al uso de la fuerza y, sobre todo, la proliferación de armas de destrucción masiva al alcance también de agentes no estatales en un mercado internacional de armamento que contradice asimismo la normativa internacional ${ }^{15}$. Esta última observación es particularmente relevante a la hora de afrontar la amenaza terrorista: el incremento y diseminación sin control de arsenales, tanto convencionales como incluso nucleares, el desarrollo y relativo fácil acceso a nuevas tecnologías, cuyo primer resultado es que actores absolutamente nuevos e incluso imprevisibles (los no estatales) encuentran la posibilidad de erigirse como gravísimas amenazas para la seguridad y la paz internacionales, más allá de las tradicionales manifestaciones del terrorismo internacional. Eso es particularmente claro por lo que se refiere a un escenario tan temible como verosímil, el de las ciberguerras en términos de nueva modalidad de amenaza terrorista, y exige una capacidad de inversión que está fuera del alcance de los presupuestos estatal-nacionales e incluso, hoy por hoy, de las disponibilidades al efecto en los presupuestos de la UE.

d'Emmanuel Macron: Une vision. Des propositions (disponible en https://www.robert-schuman.eu/fr/ questions-d-europe/0445-1-europe-d-emmanuel-macron-une-vision-des-propositions).

15 Révue Stratégique de la Défense et de la Sécurité Nationale 2017, Synthése, cit., apartado 2: «Le système international issu de la guerre froide cède la place à un environnement multipolaire en profonde mutation, dont l'instabilité et l'imprévisibilité sont les figures dominantes. L'affirmation militaire d'un nombre croissant de puissances, établies ou émergentes, dans des régions sous tension (Levant, golfe Arabo-Persique, Asie), s'accompagne de politiques de rapports de forces, voire de fait accompli. Elle nourrit également des logiques de compétition, pour l'accès aux ressources et pour le contrôle des espaces stratégiques, matériels et immatériels (maritime, aérien, espace exo-atmosphérique, espace numérique). Ces évolutions se doublent d'une contestation directe des institutions internationales et des normes censées encadrer le recours à la force, et d'une poursuite préoccupante des logiques de prolifération, des armes de destruction massive comme de leurs vecteurs. A l'appui de ces stratégies de puissance, le développement de nouveaux modes opératoires, basés sur l'ambiguiité des intentions, la combinaison de moyens d'actions - militaires ou non - à des fins d'intimidation ou de déstabilisation, comporte des risques élevés d'escalade». 
En definitiva, todas esas consideraciones ofrecen un mapa de problemas extraordinariamente complejo y, a la vez, apremiante. En todo caso, me gustaría llamar la atención precisamente sobre la dimensión de urgencia, que sugiere que está en juego la supervivencia del propio proyecto europeo. Porque podría hacer verosímil, paradójicamente, un avance sustantivo hacia el objetivo de alcanzar una política de defensa común que, reitero, se encuentra ahora inserto en el Tratado de la propia UE. De nuevo, se trata de la verosimilitud que proporciona la necesidad, que fuerza, como reza el dictum, la aparición de la virtud ${ }^{16}$.

Semejante efecto paradójico de refuerzo de la unidad de acción exterior de la UE se advierte, por ejemplo, en la reacción de la UE ante uno de los más grave y recientes despropósitos de la administración Trump. Me refiero a la posición firme y común de la UE en relación con la estrategia de la administración norteamericana acerca de la renovación de los acuerdos con Irán, bajo el argumento entre otros, de la complicidad del régimen iraní con movimientos terroristas y no sólo el bien conocido de su confirmación como potencia nuclear. En efecto, la Alta Representante, en rueda de prensa mantenida el 13 de octubre, reiteró el alineamiento sin fisuras de la UE con la legalidad internacional: « Ce n'est pas aux Etats-Unis de décider seuls de changer un deal international... Aucun acteur, aucun dirigeant dans le monde ne peut contester le contenu d'une résolution des Nations Unies adoptée à l'unanimité. Certes le président américain a de nombreux pouvoirs. Mais il n'a pas ce pouvoir-là $\gg^{17}$.

En todo caso, como decía, es posible que hoy, en otoño de 2017, las condiciones adversas que afrontamos, paradójicamente (o no tanto) estimulen lo impensable, un desarrollo de ese proyecto autónomo europeo de seguridad y defensa. Y lo cierto es que en estos meses se han producido pasos que deben considerarse significativos, como veremos en la primera parte del epígrafe siguiente.

16 Coincido con el planteamiento que propone el título de la contribución del eurodiputado socialista JAÚREGUI, R., «La defensa europea tras el Brexit, o cómo hacer de la necesidad virtud», en el monográfico del $\mathrm{n}^{\circ} 275$ de la revista Temas, mencionado en la nota 1 . Sin embargo, creo que el enfoque de Jaúregui incurre en el error justamente denunciado por Pascale Jeannin, esto es, insistir en la dimensión instrumental, la «Europa de la defensa», en lugar de en el objetivo básico, la «defensa de Europa», tal y como señalaré. En definitiva, como también apuntaré, en línea con JEAnNIN, P., «La défense de l'Europe, avant l'Europe de la défense», Cuestions de l'Europe, $\mathrm{n}^{\circ} 377,11 / 01 / 2016$, Foundation Robert Schuman, al abandono de lo que podría calificarse como el «método Schuman».

17 Cfr. «Les européens entament un bras de fer avec Washington». Bruxelles 2. https://www.bruxelles2.eu/2017/10/16/une-certaine-victoire-europeenne/ (Consultado el 16/9/2017). 
3. Principales hitos de la eVolución de la estrategia global en 2017. ESPECÍFICAMENTE EN MATERIA DE LUCHA ANTITERRORISTA

En lo que sigue, voy a referirme en primer lugar (apartado 3.1), de forma sumaria, a los principales acontecimientos que permitirían hablar de avances en la consolidación de la Estrategia Global de seguridad y defensa europeas, para, a continuación (apartado 3.2.), examinar con mayor detalle los principales elementos que atañen específicamente a la estrategia europea en materia de la lucha antiterrorista ${ }^{18}$. Entre otros, el Consejo Europeo de junio de 2017 ${ }^{19}$, que fue precedido por el Consejo de Asuntos Exteriores específicamente dedicado a estrategia de lucha antiterrorista ${ }^{20}$, el Consejo Europeo JAI de 14 de septiembre de $2017^{21}$ y sobre todo, la Directiva europea de 2017 relativa a la lucha contra el terrorismo ${ }^{22}$. Todo eso debe ser completado, por la importancia del eje franco-alemás, con el examen de las principales características del modelo antiterrorista adoptado por Francia y el debate acera de ese particular en la RFA, como veremos en el epígrafe siguiente (4).

\subsection{El desarrollo de la estrategia global de seguridad y defensa en 2017}

Si alguien ha puesto el dedo en la llaga de una cuestión básica para el desarrollo de la política europea de seguridad y defensa ha sido el Vicepresidente de la UE Jirki Kataninen, responsable de Inversiones, Crecimiento y Competitividad, y muy específicamente responsable del nuevo desarrollo del nuevo Fondo Europeo de Defensa. En efecto, en n una reciente entrevista en Reuters $^{23}$, poco antes del Consejo Europeo de 22 y 23 de junio de 2017, Kataninen se mostraba escéptico sobre la política de seguridad y defensa de la UE y, por tanto, sobre los avances en la Estrategia Global, en la medida en que el esfuerzo presupuestario no es suficiente para abordar los objetivos trazados. «Los Estados Miembros se han dado cuenta de que la situación actual es in-

18 Una información completa sobre las iniciativas, documentos y resoluciones de la UE en materia de la estrategia antiterrorista puede encontrarse en el sito web del Consejo Europeo, http:// www.consilium.europa.eu/es/policies/fight-against-terrorism/.

19 Cfr. http://www.consilium.europa.eu/es/press/press-releases/2017/06/23-euco-conclusions/.

20 Cfr. http://www.consilium.europa.eu/es/press/press-releases/2017/06/19-conclusions-counterterrorism/

21 Cfr. http://www.consilium.europa.eu/es/meetings/jha/2017/09/14/.

22 Cfr. http://eur-lex.europea.eu/legal-content/ES/TXT/?uri=CELEX\%3A32017LO541

23 Publicada el 11 de junio de 2017, en el diario El Mundo. 
sostenible. Hay amenazas convencionales, híbridas, ataques terroristas, cuestiones relacionadas con las migraciones, ciberataques. No hay forma de que sigamos como hasta ahora, y no basta siquiera invertir el 2\% del PIB a menos que se haga algo diferente... Se trata de gastar más, sí, pero también mejor».

Los números cantan, añade el vicepresidente: «El coste anual de las duplicidades en la UE en temas de Defensa es de 25.000 a 100.000 millones. El 80\% de las compras se hacen en función de necesidades nacionales, al igual que el $90 \%$ de la I+D militar. Si lo hiciésemos bien ahorraríamos el 30\% anual. La UE se gasta el 50\% de lo que hace EEUU. La UE invierte 28.000 euros por soldado, teniendo todo en cuenta, formación, equipo e investigación y desarrollo, mientras que EEUU, 108.000». Y por eso propone un cambio: «Dedicar 500 millones al año a I+D y 5.000 para el desarrollo de las capacidades. De esos 5.000, queremos que 1.000 millones salgan del Presupuesto europeo y el resto, de los Estados».

Por ahora estas propuestas no han encontrado concreción. El Consejo Europeo de diciembre de 2016 ratificó tres prioridades estratégicas que no han sido modificadas sustancialmente en el Consejo europeo de junio de 2017. Estas son:

(1) Dar respuesta a los conflictos y crisis exteriores

(2) Aumentar las capacidades de los socios

(3) Proteger a la UE y sus ciudadanos.

Entre las medidas concretas para alcanzar esos objetivos, se proponen estas cuatro:

- La puesta en marcha de una revisión anual coordinada de la defensa para mejorara la cooperación en materia de defensa entre los Estados miembros ${ }^{24}$.

- El establecimiento de una cooperación estructurada permanente (PESCO) para reforzar la cooperación en materia de defensa entre aquellos Estados miembros que deseen intensificarla ${ }^{25}$.

24 Se trataría de ofrecer una mejor visión general del gasto, las inversiones nacionales y el esfuerzo de investigación de la UE en defensa y, junto a ello, aumentar la transparencia y la visibilidad política de las capacidades de defensa europeas. De esa forma, se conseguiría una mejora de la detección de carencias, se intensificaría la cooperación en materia de defensa y, en definitiva, se conseguiría un enfoque mejorado y más coherente de la planificación del gasto en defensa

25 En efecto, el Consejo acordó poner en marcha una cooperación estructurada permanente con objeto de reforzar la seguridad y la defensa de Europa. A esos efectos, se estableció la previsión de que los Estados miembros acuerden una lista común de criterios y compromisos, junto con proyectos concretos de capacidades, con el fin de iniciar esta cooperación. 
- La creación de una Capacidad Militar de Planificación y Ejecución dentro del Estado Mayor de la UE, para mejorar las estructuras de gestión de crisis y la capacidad de la UE para reaccionar de forma más rápida, eficaz y coherente, acordada en el Consejo de Ministros de defensa de 8 de junio de 2017.

- El refuerzo del conjunto de instrumentos de respuesta rápida de la UE, en particular las capacidades civiles y los grupos de combate de la UE. A ese respecto, se acordó asumir como coste común el despliegue de grupos de combate. La financiación de estos grupos se gestionará con carácter permanente a escala de la UE a través del mecanismo Athena. Se acuerda asimismo desarrollar la capacidad de respuesta de la gestión civil de crisis, en particular mediante la posible creación de una capacidad básica de respuesta.

Como decía, el Consejo Europeo de junio de 2017 no supuso particulares modificaciones en la línea enunciada respecto a la implementación y desarrollo de la Estrategia Global europea de seguridad y defensa ${ }^{26}$.

\subsection{Los avances en materia de lucha europea contra el terrorismo en 2017}

En este apartado me referiré de forma sumaria a cuatro de los instrumentos que abordan la estrategia europea antiterrorista en el año 2017 y que me parecen de mayor interés: (1) Las conclusiones del Consejo de Asuntos exteriores previo al Consejo Europeo de junio de 2017; (2) las del propio Consejo Europeo de junio de 2017; (3) las del Consejo JAI sobre terrorismo de septiembre de 2017; y finalmente (4), la Directiva europea 2017/541, de lucha contra el terrorismo de 15 de marzo de 2017, que es anterior, pero cuyo rango es el más importante en términos de los instrumentos jurídicos de dicha estrategia.

26 Sobre ello me permito de nuevo la remisión al análisis que propuse en el trabajo mencionado en la nota 5. En ese trabajo he llamado la atención sobre lo que considero un riesgo de efectos potencialmente perversos, la inclusión del control de los movimientos migratorios (y de refugiados) como parte integrante de esta estrategia global de seguridad y defensa. Así lo abordan las conclusiones del Consejo de junio de 2017, en su apartado IV (epígrafes 20 a 23), si bien es cierto que se destaca la preocupación por la pérdida de vidas en el Mediterráneo y la necesidad de desarrollar «la cooperación con los países de origen y de tránsito, incluido el fortalecimiento de la cooperación regional en actividades de búsqueda y salvamento, que sigue siendo una de las principales prioridades». La insistencia, sin embargo, en el papel de Libia - un Estado fallido- es uno de los puntos negros de esta estrategia europea, como también señalé en mencionado trabajo. 
3.2.1. Las Conclusiones del Consejo de Asuntos Exteriores de 19 de junio de 2017, en materia de lucha contra el terrorismo, previas al Consejo Europeo de 22 y 23 de junio UE.

Este Consejo Europeo de Asuntos Exteriores dedicado específicamente a la estrategia europea de lucha antiterrorista en su dimensión internacional, parte de reafirmar que las Conclusiones sobre lucha contra el terrorismo adoptadas el 9 de febrero de 2015, a raíz de los atentados contra Charlie Hebdo (enero de 2015), siguen constituyendo la piedra angular de la acción exterior de la UE en la lucha contra el terrorismo, aunque reconoce expresamente que la UE ha avanzado significativamente en este ámbito en escasamente dos años en este ámbito.

En sus Conclusiones, destaca la necesidad de reforzar y desarrollar las estructuras de lucha contra el terrorismo, a fin de fortalecer la capacidad de la UE para intensificar la cooperación en la lucha antiterrorista. Pone de relieve el vínculo entre la dimensión interior y la exterior de esta estrategia de lucha, a fin de asegurar una mayor coherencia entre las acciones a nivel interior y exterior en el ámbito de la seguridad, reforzando el papel de las agencias de la JAI respecto de terceros países: en particular, en las delegaciones exteriores de la UE mediante expertos en lucha contra el terrorismo y seguridad. El Consejo observa que con la inclusión, mediante las Conclusiones del Consejo de mayo de 2017, de la lucha contra el terrorismo en las misiones de Feira, las misiones y operaciones de la PCSD desempeñan ahora un papel reforzado en la lucha contra el terrorismo; el refuerzo de la cooperación con Oriente Próximo y el Norte de África, los Balcanes Occidentales, Turquía, el Sahel y el Cuerno de África, mediante un diálogo político más intenso, más proyectos de lucha contra el terrorismo y apoyo financiero a la lucha contra el terrorismo y a la prevención y lucha contra el extremismo violento, y el refuerzo de la comunicaciones estratégicas, en particular del Grupo Operativo Sur de la División de Comunicaciones Estratégicas; el refuerzo de la cooperación internacional, en particular con socios estratégicos fundamentales como los Estados Unidos, Australia, Canadá y los socios de Schengen, así como con organismos regionales y multilaterales como las Naciones Unidas, la OTAN, el Foro Mundial contra el Terrorismo, Interpol y la coalición global contra el Daesh; el refuerzo de la respuesta de la UE en ámbitos temáticos esenciales, como la prevención y lucha contra el extremismo violento, la necesidad de abordar de forma eficaz la captación y radicalización en línea, el grave desafío que constituyen los combatientes terroristas extranjeros, en particular la cuestión de los retornados, la seguridad de la aviación, el tráfico de armas de fuego, la cuestión de la 
financiación del terrorismo y el blanqueo de capitales, así como los vínculos entre la delincuencia grave y organizada y el terrorismo.

3.2.2. En el Consejo Europeo de 22 y 23 de junio de 2017 se abordó la revisión y prospectiva de la estrategia europea de lucha antiterrorista, en el marco de la ya mencionada Estrategia Global. A ese respecto, en sus Conclusiones se señala expresamente lo que como hemos visto, con Jeannin, cabe denominar el método Schuman, es decir, la protección de los ciudadanos europeos como perspectiva básica en tal estrategia. Y así, se indica:

«En el día de hoy, el Consejo Europeo ha dedicado su atención al fortalecimiento de Europa y a la protección de los ciudadanos mediante medidas eficaces destinadas a luchar contra el terrorismo y a desarrollar su seguridad y defensa comunes...Una Unión fuerte y resuelta es la mejor manera de fomentar nuestros valores e intereses...»

Entre las medidas que deben priorizarse en esta estrategia se concretan las siguientes:

- combatir la propagación de la radicalización en internet,

- coordinar los trabajos para prevenir y combatir el extremismo violento y hacer frente a la ideología afín

- atajar la financiación del terrorismo,

- facilitar intercambios de información ágiles y bien definidos entre las autoridades policiales, en particular con los socios de confianza,

- mejorar la interoperabilidad entre las bases de datos.»

Consideración especial merece, como ya había señalado el documento de Estrategia Global de la Alta Representante Mogherini, la lucha contra el ciber terrorismo, en donde se advierte en primer lugar de la responsabilidad que atañe a la industria de la telecomunicación y a las redes, clave en la tarea de detección y prevención de ataques terroristas y no sólo de los cibernéticos:

$\ll$ El sector tiene su propia responsabilidad para ayudar a combatir el terrorismo y la delincuencia en red. Partiendo de la labor que realiza el Foro de la Unión Europea sobre Internet, el Consejo Europeo espera que el sector cree un foro sectorial y desarrolle nuevas tecnologías y herramientas para mejorar la detección automática de contenidos y eliminar aquellos que inciten a perpetrar actos de terrorismo. Esto deberá completarse, en caso necesario, con las correspondientes medidas legislativas a escala de la UE. Pide que se aborden los retos planteados por los sistemas que permiten a los terroristas comunicarse sin que las autoridades competentes puedan acceder a esa comunicación...» 
3.2.3. Finalmente, el Consejo de Justicia e Interior celebrado el pasado 14 de septiembre de $2017^{27}$ abordó las peticiones de un grupo de países de la UE que exigen una prolongación en las medidas de controles excepcionales fronterizos previstos en el Código Schengen, con el argumento de que así lo exige la respuesta a la amenaza terrorista que sufre la Unión Europea.

Conviene recordar que, en la actualidad, aplican controles en las fronteras internas seis países del espacio Schengen: Francia, Alemania, Austria, Dinamarca, Suecia y Noruega. Francia lo hace desde 2015, tras los atentados de noviembre en París, mientras que los otros cinco países los aplican desde 2016 para responder a los movimientos migratorios tras la llamada «crisis de los refugiados». Los países que han insistido más en la necesidad de nueva prolongación, Francia y Alemania, reclamaban ya desde el pasado febrero cambios en la legislación para reforzar la seguridad en Europa y, en particular, en el Código Schengen. Junto a ellos, Austria y Noruega, proponen prolongar de dos a cuatro años los controles excepcionales en frontera previstos en el Código Schengen bajo el amparo de la amenaza terrorista. Estos países plantean, en particular, una reforma del artículo 25 del Código Schengen ${ }^{28}$. Los firmantes del documento piden a la Comisión Europea que presente normas para modificar ese artículo y permitir a los Estados miembros «reintroducir controles en las fronteras interiores por periodos más largos que los actualmente previstos». Consideran que la limitación a seis meses para la reintroducción de los controles por motivo de una grave amenaza pública o a la seguridad interna «no se corresponde con las necesidades en el contexto de una amenaza terrorista a largo plazo». Argumentan que los controles -adoptados después de que más de un millón de personas consiguieran cruzar las fronteras europeas en busca de asilo en 2015- y que terminan en noviembre de 2017, son necesarios por razones de seguridad ${ }^{29}$.

En ese Consejo, el Comisario europeo de Interior, Dimitris Avramapoulos, apoyó el argumento de estos Estados miembros, pero no propuso su adopción como tal, porque respaldaba la posición oficial de que los controles introducidos «deben finalizar» en noviembre de 2017, dado que las principales razones para la prolongación de los mismos «ya no existen». Pese a ello, respaldó la necesi-

27 Cfr. http://www.consilium.europa.eu/es/meetings/jha/2017/09/14/.

28 Las normas actuales prevén que los países puedan restablecer los controles fronterizos por un periodo inicial de 30 días, ampliable hasta seis meses, prorrogables hasta alcanzar un máximo de dos años, en casos excepcionales.

29 Recordemos que el Consejo Europeo autorizó a todos esos países la prolongación de los controles, supuestamente por última vez, en mayo de 2017, hasta noviembre de 2017. 
dad de cambiar las reglas que permiten a los europeos moverse entre países sin la necesidad de mostrar su documento de identidad o pasaporte. Así, admitió la necesidad de adaptar las normas a los nuevos «retos» de seguridad. Expresamente, haciéndose eco de los dos más recientes atentados (Cataluña, Finlandia) el Comisario reconoció que «han surgido nuevos retos para la seguridad en los últimos años, como demuestran los atentados más recientes en Catalunya y Turku. Así pues, Avramopoulos admitió que el sistema Schengen puede «no estar suficientemente adaptado para responder a esos desafíos», razón por la cual el avanzó que la Comisión Europea «presentará un paquete para reforzar Schengen», que incluirá una propuesta para poner al día esa legislación.

Particularmente significativa fue la toma de postura del representante del Gobierno francés, el ministro del Interior, Gérard Collomb, quien subrayó en declaraciones a la prensa la importancia de «tener medios para seguir controlando las fronteras», teniendo en cuenta que «el terrorismo es «transfronterizo» y que algunos de los responsables de los últimos atentados cometidos en suelo europeo habían circulado entre varios Estados miembros (Francia, Bélgica, España). En ese contexto, indicó que tras la reunión tiene la impresión de que el comisario «desea suavizar el Código, sin poner en peligro la libre circulación, para permitir proteger las fronteras contra el terrorismo». Collomb recordó que en teoría los controles fronterizos que se aplican ahora en algunos países finalizan en noviembre y que la idea sería «poder seguir un poco más de tiempo», una idea que aseguró es «apoyada por muchos Estados» y que confió en que tendrá «una acogida favorable».

3.2.4. El documento más relevante en materia de la estrategia de lucha antiterrorista adoptado en 2017 es, sin duda, la Directiva 2017/541 de 15 de marzo de 2017 relativa a la lucha contra el terrorismo ${ }^{30}$.

Los críticos de esta norma han destacado el lenguaje bélico, que parece encuadrarse más bien en la estrategia de «guerra contra el terrorismo» propia de las administraciones norteamericanas desde que se aprueba la Patriot Act,

30 Cfr. Directiva UE 2017/541 de 15 de marzo de 2017, relativa a la lucha contra el terrorismo y por la que se sustituye la Decisión marco 2002/475/JAI del Consejo y se modifica la Decisión 2005/671/JAI del Consejo. http://eur-lex.europa.eu/legal-content/ES/TXT/PDF/?uri=CELE X:32017L0541\&from=ES. Es importante señalar que en su artículo 28.1, relativo al plazo de trasposición de la Directiva, se señala: «Los Estados miembros pondrán en vigor las disposiciones legales, reglamentarias y administrativas necesarias para dar cumplimiento a lo establecido en la presente Directiva a más tardar el 8 de septiembre de 2018». 
tras los atentados terroristas de septiembre de 2001 y no en lo que se consideraba la especificidad de la lucha europea contra el terrorismo, que prima los instrumentos propios del Estado de Derecho (no del estado de guerra), esto es el trabajo de inteligencia y policial y la actuación del poder judicial. Lo cierto es que esa línea quiebra primero en el Reino Unido y, sobre todo, en Francia tras los atentados de Paris de noviembre de 2015. El Gobierno del Presidente Hollande adoptó una línea de respuesta que recurre al estado de alarma en todo el territorio y que incluye acciones de guerra contra las bases del ISIS, considerado responsable de los ataques. Francia se considera abierta y manifiestamente en guerra contra el terrorismo internacional. En ese sentido, puede entenderse la razón de que la directiva se exprese en términos como los siguientes: «Los denominados 'combatientes terroristas extranjeros' viajan al extranjero con fines terroristas... han estado relacionados con atentados y complots recientes en varios Estados miembros. Además, la Unión y sus Estados miembros se enfrentan a la creciente amenaza que representan las personas que, aunque permanezcan dentro de Europa, reciben inspiración o instrucciones de grupos terroristas situados en el extranjero». Del mismo modo, cuando la directiva aborda la necesidad de coordinar entre los Estados miembros la tipificación de los delitos de terrorismo ${ }^{31}$, con especial atención

31 Las conductas contemplados como delitos graves son «Los atentados contra la vida de las personas, en tanto actos intencionados que pueden calificarse de delitos de terrorismo cuando y en la medida en que se cometan con un fin terrorista específico, a saber: intimidar gravemente a la población, presionar indebidamente a los poderes públicos o a una organización internacional para que lleve a cabo o se abstenga de llevar a cabo cualquier acto, o desestabilizar gravemente o destruir las estructuras políticas, constitucionales, económicas o sociales fundamentales de un país o de una organización internacional. También se debe considerar delito de terrorismo la amenaza de cometer esos actos intencionados cuando quede establecido, sobre la base de circunstancias objetivas, que tal amenaza se produjo con cualquiera de esos fines terroristas...». No son los únicos delitos que se contemplan en la Directiva. Así, se afirma, «Los delitos relacionados con las actividades terroristas son de extrema gravedad, ya que pueden llevar a la comisión de delitos de terrorismo y permitir que los terroristas y los grupos terroristas mantengan y sigan desarrollando sus actividades delictivas, lo que justifica la tipificación penal de dicha conducta». Y se añade que entre los delitos de provocación pública a la comisión de un delito de terrorismo se han de incluir «la apología y la justificación del terrorismo o la difusión de mensajes o imágenes, ya sea en línea o no, entre ellas las relacionadas con las víctimas del terrorismo, con objeto de obtener apoyo para causas terroristas o de intimidar gravemente a la población. Esta conducta debe tipificarse cuando conlleve el riesgo de que puedan cometerse actos terroristas. En cada caso concreto, al examinar si se ha materializado ese riesgo se deben tener en cuenta las circunstancias específicas del caso, como el autor y el destinatario del mensaje, así como el contexto en el que se haya cometido el acto. También deben considerarse la importancia y la verosimilitud del riesgo al aplicar la disposición sobre provocación pública de acuerdo con el Derecho nacional». 
a la financiación de las actividades terroristas, se insiste en el concepto de «combatientes terroristas extranjeros», cuyas conductas deben ser castigadas también si se cometen a través de internet, incluidas las redes sociales».

Particularmente discutibles resultan dos aspectos de esta tipificación coordinada de los delitos de terrorismo. Me refiero en primer lugar a algunas de las conductas que se engloban bajo el denominador de «adiestramiento en terrorismo». En efecto, señala la directiva: «La tipificación penal de la recepción de adiestramiento para el terrorismo complementa el delito ya reconocido de adiestramiento y aborda específicamente las amenazas que plantean las personas que se preparan activamente para la comisión de delitos de terrorismo, entre ellas las que acaban actuando en solitario. La recepción de adiestramiento para el terrorismo incluye la obtención de conocimientos, documentación o capacidades prácticas. El aprendizaje autónomo, en particular a través de internet o consultando otro tipo de material de aprendizaje, también debe considerarse recepción de adiestramiento para el terrorismo cuando sea el resultado de una conducta activa y se efectúe con la intención de cometer o contribuir a la comisión de un delito de terrorismo. En el contexto de todas las circunstancias específicas del caso, esta intención puede inferirse, por ejemplo, del tipo de materiales y de la frecuencia de la consulta. Por lo tanto, descargarse un manual para fabricar explosivos con el fin de cometer un delito de terrorismo podría considerarse recepción de adiestramiento para el terrorismo». El problema consiste en distinguir perfectamente lo que es la lectura de un lugar en la web en el que se habla de esos materiales y precisar la no concurrencia de intención, sobre todo por la indeterminación de la expresión «consulta frecuente».

En segundo lugar, la tipificación de una figura que preocupa enormemente, los viajes al extranjero con fines de adiestramiento o participación en actividades terroristas. La UE es consciente de que la inmensa mayoría de los atentados terroristas son cometidos por ciudadanos europeos o con residencia legal estable en Estados miembros, de modo que el peligro no reside tanto en la llegada de extranjeros, como a veces se asegura contra toda evidencia, para a continuación postular políticas de cierre de fronteras. Pues bien, a este propósito se afirma: «En vista de la gravedad de la amenaza y de necesidad, en particular, de frenar el flujo de combatientes terroristas extranjeros, es preciso tipificar el hecho de viajar al extranjero con fines terroristas... No es indispensable tipificar el acto de viajar como tal... Debe asimismo tipificarse cualquier acto que facilite tales viajes». Ese grado de indeterminación resulta como mínimo criticable.

Menos polémica es la insistencia en la necesidad de coordinación internacional y no sólo europea, habida cuenta del «carácter transfronterizo del 
terrorismo», que exige «una firme y coordinada respuesta y una firme cooperación en y entre los Estados miembros así como con y entre las agencias y órganos competentes de la Unión para combatir el terrorismo, entre otros Eurojust y Europol». Aún más, se añade, «El carácter mundial del terrorismo requiere una respuesta internacional, lo que exige que la Unión y sus Estados miembros refuercen la cooperación con los terceros países pertinentes».

La directiva presta atención al comercio ilícito de armas como amenaza creciente en materia de seguridad y vinculada a la financiación del terrorismo, y asimismo pone énfasis en la actividad de prevención, en la que destaca el trabajo contra los procesos de radicalización que, señala, «requiere un planteamiento global y preventivo a largo plazo», lo que supone «combinar medidas en materia de justicia penal con políticas en materia de educación, inclusión social e integración, así como la disponibilidad de programas efectivos de desradicalización o desmovilización, de salida o rehabilitación». Los trabajos de «coordinación y el intercambio de información y experiencias sobre políticas nacionales de prevención» son considerados prioritarios, así como las «medidas de formación y de sensibilización destinadas a facilitar la detección de los signos de radicalización y a hacerles frente»

\section{Sobre la estrategia antiterrorista del eje Franco-Alemán}

Como hemos visto, el desarrollo de la Agenda global sobre seguridad y defensa depende en buena medida de encontrar un núcleo que impulse con determinación ese proyecto de defensa de Europa, lo que a la vista del contexto europeo parece empresa difícil. Sin embargo, en lo que se refiere al desarrollo específico de la estrategia de lucha contra el terrorismo, es evidente que la dificultad es mucho menor en la medida en que se trata de combatir una amenaza real y global, en la que la coordinación de recursos es capital.

En este ámbito, el realismo obliga a reconocer que en lo que se refiere al avance de una estrategia europea de seguridad y defensa no esté tan claro que podemos contar con los candidatos 'naturales' para ese liderazgo, Francia, Alemania y el Reino Unido. Alemania, en este aspecto, está fuertemente limitada por sus disposiciones constitucionales. Es más, tras los resultados de las elecciones legislativas de 2017 , la minoría en que se encuentra la primera fuerza, la CDU/CSU de la canciller Merkel, ante la negativa además de repetir la Grosse Koalition con el SPD, parece abocada a lo que se denomina ya una «coalición Jamaica», con liberales y verdes. Pero el hecho es que ni unos ni 
otros aparecen como fervientes europeístas ni, menos aún, como partidarios de apoyar el refuerzo de una Europa de la defensa. Por su parte, el Reino Unido no parece poder cumplir ese papel de liderazgo, porque con el Brexit acaba de decidir dejar la UE, por más que no quiera, ni deba, ni pueda, desvincularse del objetivo de defensa y seguridad europea, en particular frente a la primera y más grave amenaza, el terrorismo internacional. Es obvio que el Reino Unido permanece como uno de los pilares de la OTAN y conserva su condición de aliado privilegiado de los EEUU, factores que han de tenerse en cuenta a la hora de rediseñar una PCSD sin su presencia. Pero no cabe esperar su iniciativa.

Con todo, no cabe desdeñar la tesis de quienes sostienen que la constatación de semejantes obviedades no debería hacer valer sólo las dificultades, sino que es una oportunidad para replantear desde otra perspectiva la propia PCSD, explorando las posibilidades que ofrece la cooperación estructural permanente (PESCO) y sobre todo la lógica de la Europa de dos velocidades también a propósito de seguridad y defensa, con Estados miembros decididos a avanzar más adelante en una política común ${ }^{32}$.

De cualquier forma, como ya he señalado, eso no debiera impedirnos reconocer los signos positivos que llegan desde la única potencia nuclear de la UE que, además es el único miembro de la UE en el Consejo de Seguridad, Francia. Y a este respecto no se puede minusvalorar la expresa y decidida voluntad política del Gobierno francés presidido por Emmanuel Macron, de impulsar el proyecto europeo y, en su seno, la PCSD. No se trata sólo de la

32 Así lo apuntan Dumoulin, A. y Gros-Verheyde, N., La politique européenne de sécurité et de défense commune (Parce que l'Europe vaut bien une défense), ed. Du Villard, Bruxelles, 2017, p. 463, cuando escriben: «Il s'agit maintenant de réformer l'Union en saississant la porte ouverte par le Brexit. L'Europe à deux vitesses, en cercles concentriques, ou différenciée, apparaît ainsi comme le modèle permettant d'avancer, pour ceux qui le souhaitent, et de ne pas être laissés de côté, pour tous les autres. Le Traité de Lisbonne contient les avancées nécessaires en matière de défense européenne. Les briques constitutives d'une véritable autonomie en matière de sécurité et de défense sont là. La coopération structurée permanente permet à quelques États plus déterminés, d'aller plus loin au niveau opérationnel comme capacitaire, pour partager et gérer en commun certains moyens, comme pour intervenir ensemble, de façaon plus efficce ou plus robuste, avec des objectifs contraignants. Le niveau d'ambition conditionnera la pertinence et la crédibilité à venir des Européens en matière de sécurité et de défense. Cette coopération structurée a un avantage (et un défaut) : elle est unique. Si elle s'élargit, elle perd son âme. Si elle reste fondée sur trop peu de pays, elle nuit à la cohésion européenne. Il faudra donc la compléter par des coopérations renforcées, $a d$ hoc, plus souples, à la carte, permettant autour du noyau dur d'avoir une constellation satellitaire plus large, et de trouver les moyens de relier la coopération structurée permanente et les coopérations renforcées pour en faire un dispositif plus cohérent, sans trop de contrainte administrative». 
retórica de una nueva Europa, tal y como lo propone Macron en su ya mencionado discurso sobre una Europa soberana, unida y democrática, en el que Europa aparece como una necesidad, como la única alternativa deseable ${ }^{33}$. Se trata de un compromiso firme que subraya que el objetivo debe ser «la capacidad de acción autónoma de Europa, complementaria de la OTAN», y con aspectos muy concretos en lo que se refiere a la defensa europea, desde la base de una cultura estratégica común. Así, se establecen como propuestas para 2020 una fuerza común de intervención, un presupuesto de defensa común, una doctrina común de intervención, la puesta en marcha de un Fondo europeo de defensa, la Cooperación estructural permanente, o la creación de una Academia europea de inteligencia.

Todo ello viene confirmado en el mencionado documento de estrategia de defensa francesa de 2017, coordinado por el eurodiputado Arnaud Danjean, donde Macron inscribe en su prefacio esta solemne declaración:

«Enfin, les progrès de l'Europe dans le domaine de la défense devront être consolidés. Les bases d'une autonomie stratégique européenne ont été posées. Plusieurs de nos partenaires prennent conscience que l'Europe constitue, dans le cadre d'une relation transatlantique équilibrée, le cadre naturel de notre sécurité, de la protection de nos frontières, face à des défis qui ne peuvent être relevés qu'ensemble. Le moment est donc venu de relancer l'Europe de la défense, en rapprochant nos cultures stratégiques, en nourrissant des partenariats pragmatiques avec les Etats européens qui ont la volonté politique et la capacité militaire d'assumer avec nous leurs responsabilités en opérations, en dégageant les ressources nécessaires au niveau européen, en consolidant nos industries de défense pour qu'elles conservent leur excellence technologique et demeurent compétitives à l'échelle mondiale».

Podemos advertir, como subraya J.D. Giulani ${ }^{34}$, que se reafirma con claridad que Francia comparte las dos notas del documento de Estrategia de

33 «Regardez notre époque (...) et vous verrez que vous n'avez pas le choix, vous n'avez pas le luxe de la génération qui nous a précédés et qui a pu gérer ce qui était acquis et à peine construit. Vous n'avez qu'un choix simple, celui de laisser un peu plus de place à chaque élection aux nationalistes, à ceux qui détestent l'Europe et, dans cinq ans, dans dix ans, dans quinze ans, ils seront là. Nous les avons déjà vus gagner ici».

34 Cfr. GiUliani, J.D., 2017, «Une stratégie française pour la défense de l'Europe», http://www. jd-giuliani.eu/fr/article/cat-2/564_Une-strategie-francaise-pour-la-defense-de-l-Europe.html 
Mogherini, que definen la PSCD. De un lado, la seguridad y defensa europeas tienen una dimensión global y, por ello, la importancia de ampliar el radio de acción de la defensa ${ }^{35}$. Pero sobre todo la defensa europea se basa en los intereses, necesidades y valores compartidos por los europeos y por ello es una defensa que debe reafirmar su dimensión de autonomía, una Europa sólida en torno a una estrategia autónoma, propia, para ser auténticamente europea, aunque Macron es partidario de un núcleo de vanguardia de Estados europeos que tomen la tarea de impulsar esa política común, a partir de Francia, Alemania, Italia y España. Las propuestas del joven presidente francés en materia de política de seguridad y defensa incluyen la creación de una fuerza común de intervención rápida, una fiscalía antiterrorista europea, una policía europea de fronteras, una agencia única para los solicitantes de asilo y, sobre todo, un presupuesto común para la defensa.

\section{Sobre loS PRINCIPIOS Y BUENAS PRÁCTICAS EN LA ESTRATEGIA ANTITERRORISTA, COMO PRIORIDAD DE LA POLÍTICA DE SEGURIDAD Y DEFENSA EUROPEAS}

Hemos reiterado a lo largo de estas páginas que hoy, la prioridad de la estrategia europea de seguridad y defensa común es la lucha europea contra el terrorismo, que se inserta, sí, en una estrategia global, pero con acentos propios, sobre todo respecto a los que caracterizan la «guerra contra el terrorismo» desplegada por los EEUU. Sin duda, los esfuerzos fundamentales han de centrarse en sostener de forma coherente lo que constituye la razón de ser de una estrategia contra el terrorismo, esto es, la defensa del Estado de Derecho y de la democracia, lo que, en términos europeos es tanto como decir que la prioridad es ese espacio de justicia, libertad y seguridad comunes que encuentra su sentido en la garantía de los derechos y libertades fundamentales.

35 Giuliani insiste en la importancia del espacio marítimo ante las nuevas exigencias globales de seguridad y defensa, y, así, escribe: «Pour l'Europe, comme pour la France, la défense commence au loin, au fond de tous les océans, où passent les câbles sous-marins acheminant notre trafic informationnel, sur toutes les mers qui portent nos exportations et nos approvisionnements et dans toutes les régions de la planète où s'expriment de nouvelles stratégies d'intimidation, la remise en cause de principes fondamentaux comme la liberté de navigation, des tactiques d'interdiction et la volonté de créer des sphères d'influence, des agressions dissimulées utilisant de nouvelles formes de guerre ambiguë, dans l'espace ou le monde cyber». 
Cualquier iniciativa que suponga una erosión de éstos, beneficia el objetivo perseguido por el terrorismo.

En ese sentido, me permito llamar la atención sobre los efectos negativos que pueden derivar de seguir por ejemplo el modelo que el Presidente Macron acaba de imponer en Francia y que, para muchos, supone el riesgo de una normalización del estado de excepción. Como se recordará, con ocasión de los atentados en Paris en noviembre de 2015 que causaron ciento treinta muertos, el 13 de noviembre de 2015 el Gobierno francés presidido por Hollande dictó el «estado de urgencia» que fue prorrogado por Hollande en varias ocasiones y luego por Macron, quien puso todo su empeño en la aprobación de la Loi renforçant la securité intérieure et la lutte contre le terrorisme, que entró en vigor el 3 de octubre de 2017, criticada muy duramente desde diversas instancias, como denunció Edwy Plenel en su artículo «Cuando se silencia la libertad» ${ }^{36}$ y ha explicado con detalle el profesor Fernando Flores ${ }^{37}$.

Dicho esto, el primer objetivo en la estrategia antiterrorista europea ha de ser desarrollar y fortalecer un sistema de inteligencia europea antiterrorista, y asegurar la cooperación eficaz de los diferentes sistemas de inteligencia, integrados. Eso ha de incluir la inteligencia militar y debe poner énfasis en la comunicación eficaz de todos los cuerpos policiales, no sólo los estatales, también los regionales y municipales. Los think tanks e Institutos académicos de investigación especializados en materia de terrorismo deben ser tenidos en cuenta y, en lo posible, coordinados entre sí y con los centros responsables de la estrategia europea.

En cuanto a las líneas de actuación, hay que recordar que ya el 30.11.2005 el Presidente y Coordinador del Grupo europeo de lucha contra el terrorismo dirigió una propuesta al Consejo (documento 14469/4/05), con cuatro ideas guía del denominado «compromiso estratégico» de la UE en su lucha contra el terrorismo: Prevenir, proteger, perseguir, responder. A mi juicio, siguen teniendo validez y por ese orden. Insisto, el énfasis no ha de ponerse tanto en las medidas de respuesta (así, en reformas de códigos

36 Cfr. Plentel, E., «Cuando se silencia la libertad», https://www.infolibre.es/noticias/lo_mejor_mediapart/2017/10/08/cuando_silencia_libertad_70398_1044.html?utm_source=twitter. com\&utm_medium $=$ smmshare $\& u t m \_c a m p a i g n=$ noticias\&rnot $=1026865$

37 Cfr. Flores, F., «Francia, el estado de excepción normalizado», https://www.infolibre.es/noticias/opinion/blogs/foro_milicia_democracia/2017/10/19/francia_estado_excepcion_normalizado_70819_1861.html?utm_source=twitter.com\&utm_medium=smmshare\&utm_campaign= noticias\&rnot $=1052866$. 
penales, puesto que se consideran suficientes los actuales tipos penales), sino en el esfuerzo de prevención a medio y largo plazo. El terrorismo excede fronteras, por lo que la prevención en materia de cooperación policial europea en tareas de inteligencia es la tarea fundamental, de donde la imperiosa exigencia de integración.

Respecto al marco jurídico común, esto es, al modelo de integración, debe partirse, como vimos, de lo que se establece en la reciente Directiva (UE) 2017/541 del Parlamento Europeo y del Consejo de fecha 15 de marzo de 2017-09-15 relativa a la lucha contra el terrorismo (por la que se sustituye la Decisión marco 2002/475/JAI del Consejo y se modifica la Decisión 2005/671/JAI del Consejo), que fija con claridad la dimensión penal, apunta la prioridad de una estrategia eficaz en la lucha contra la financiación, señala propuestas sobre jurisdicción y enjuiciamiento ${ }^{38}$. Recuérdese asimismo que la directiva insiste en la necesidad de un marco común de protección de víctimas y de sus derechos. Es importante que las víctimas de todo tipo de terrorismo tengan un trato igualitario, que se equipare a las víctimas del terrorismo yihadista con las víctimas de los otros tipos de terrorismo que hemos padecido en España.

La estrategia antiterrorista, también en el campo del terrorismo yihadista, se juega sobre todo dentro de nuestras fronteras, en el espacio europeo, aunque obviamente la tarea de información e inteligencia exija superar ese ámbito. El cierre de fronteras no es garantía. Por supuesto, el control es necesario, pero la suspensión del espacio Schengen no es eficaz.

La coherencia de la estrategia anti terrorista con las directrices y prácticas de la política exterior forma parte de una respuesta adecuada. Un discurso que insista en nuestro valores compartidos y que choca con la práctica de alianzas y negocios con potencias dictatoriales y que incluso financian actividades terroristas o al menos contribuyen firmemente a la difusión del mensaje salafista y wahabista, es completamente contraproducente de cara a la tarea de acomodación e integración mutua de comunidades de confesión islámica en Europa.

En la lucha antiterrorista es asimismo clave es la estrategia de comunicación, que al menos obligaría a plantear dos campos: La estrategia comunicativa

38 Quizá convendría recuperar a este respecto la iniciativa formulada por España y Rumania el 18.11.2015 para la creación de un Tribunal Internacional específico contra el terrorismo, complementaria del T.P.I. 
dirigida a la opinión pública. Esto tiene que ver con la necesidad de trabajar en una cultura de defensa y seguridad que incluye crear conciencia de lo que supone la lucha antiterrorista: insistir en sus dimensiones, pero también en los medios de los que se dispone, en la necesidad de poner en valor el Estado de Derecho y en el papel de la ciudadanía. La colaboración ciudadana es esencial Pero, en segundo lugar, es decisivo el papel de medios de comunicación y redes sociales. No se trata de pedir censura, sino de un lado de contribuir a crear buenas prácticas en los medios de comunicación y, de otro, actuar en las redes. Saber tener una estrategia de actuación en las redes. 